\title{
L'information quotidienne face au Web 2.0. La stratégie multiplateforme de six quotidiens nationaux français
}

Daily Information and Web 2.0: Six French Newspapers and Their Multiplatform Content Strategies

\section{Marta Severo}

\section{OpenEdition}

\section{Journals}

Édition électronique

URL : http://journals.openedition.org/edc/5399

DOI : 10.4000/edc.5399

ISSN : 2101-0366

Éditeur

Université Lille-3

Édition imprimée

Date de publication : 1 décembre 2013

Pagination : 89-102

ISBN : 978-2-917562-10-9

ISSN : $1270-6841$

\section{Référence électronique}

Marta Severo, "L'information quotidienne face au Web 2.0. La stratégie multiplateforme de six quotidiens nationaux français », Études de communication [En ligne], 41 | 2013, mis en ligne le 01 décembre 2013, consulté le 19 avril 2019. URL : http://journals.openedition.org/edc/5399 ; DOI 10.4000/edc.5399 


\section{MARTA SEVERO \\ L'INFORMATION QUOTIDIENNE FACE AU WEB 2.0. LA STRATÉGIE MULTIPLATEFORME DE SIX QUOTIDIENS NATIONAUX FRANÇAIS}

Alors que la mort de la presse était annoncée (Pisani, 2009; Schwartzenberg, 2007) ${ }^{1}$, on assiste aujourd'hui à la multiplication de ses formes d'existence (Greer et Yan, 2011). Face à la crise du journal papier $^{2}$ et à l'émergence de nouveaux modes de consommation de l'information liés au Web 2.0 (O'Reilly, 2005 ; Pew Internet et American Life Project, 2010), les rédactions orientent leurs efforts vers de nouvelles stratégies. De plus en plus de journaux ont non seulement une version en ligne, mais proposent également leurs contenus sur d'autres supports plus facilement consultables par le lecteur : fils RSS, newsletters, réseaux sociaux et applications pour smartphone (Boczkowski, 2004a ; Deuze, 2003).

$\mathrm{Si}$, il y a quelques années, l'organisation de l'information d'un journal avait une structure bien précise et en grande partie définie

1 Le phénomène de la crise et de la disparition des journaux quotidiens a été mis en avant à plusieurs reprises pendant les dernières années (Éveno, 2008 ; Albert, 2008 ; Poulet, 2009). Voir également le site web http://newspaperdeathwatch.com/.

2 Cet article ne vise pas à réfléchir sur les questions économiques liées à l'émergence du journalisme en ligne. Nous voulons nous concentrer sur le système informationnel et sur les stratégies de contenu liées aux nouveaux supports du Web 2.0. Aussi les questions liées à la publicité et au marketing ne seront pas abordées ici. Sur ces sujets on renvoie à Attias, 2006 ; Tarlé, 2006 ; Gentzkow, 2004. 
par la nécessité de s'adapter aux caractéristiques du support papier, la question se pose aujourd'hui dans des termes radicalement nouveaux (Charon, 2010). Pour répondre à la demande des lecteurs et contrer les offres concurrentes, la rédaction doit proposer ses contenus sur de nouvelles plateformes et donc sous de nouvelles formes.

\section{Journalisme Web 2.0 et architecture de l'information}

Le passage d'un journal papier à un dispositif multiplateforme est indéniablement une transformation très profonde qui impose des changements dans les activités des journalistes (Bakker, 2012), dans le fonctionnement du journal et dans les habitudes de la rédaction (Boczkowski, 2010). Ce qui rend cette transformation très intéressante est le fait qu'elle touche à l'organisation de l'information du journal dans sa globalité, c'est-à-dire qu'elle a des effets sur la manière dont l'information est traitée et structurée par les pratiques rédactionnelles dans toutes ses différentes phases : depuis la sélection de l'information, sa transformation en actualité, l'adaptation du contenu à chaque plateforme, jusqu'à la publication dans le mode multiplateforme.

Le passage du papier au web implique en effet de confronter l'organisation de l'information du journal avec l'architecture de l'information du Web (Rosenfeld et Morville, 1998). Pour offrir ses actualités à travers un site web, sur les réseaux sociaux, via les smartphones et tablettes, les rédactions sont contraintes de transformer le système informationnel du journal en modifiant à la fois ses caractéristiques techniques et la stratégie de contenu, lequel doit être adapté au nouveau système de publication à travers le travail du journaliste.

Dans un contexte aussi complexe de réorganisation de l'information, le concept d' « architecture de l'information », tel que l'a défini Morville, pourrait être opératoire et guider la transformation des dispositifs techniques et des formes de travail. La transformation d'un journal en multiplateforme implique la création d'un nouveau système de contenu, qui doit être fondé sur une nouvelle structure de l'information. Mais la construction de celle-ci peut résulter d'un processus conscient de redéfinition de l'architecture de l'information ou s'imposer comme l'accumulation de changements successifs et conjoncturels ne répondant pas à un plan précis pensé en amont. 
Cet article vise à étudier si et comment les stratégies de contenu d'un journal changent face à l'émergence du Web 2.0 et à l'exigence qu'elle impose de proposer la même information sur plusieurs plateformes. Comment l'information elle-même est-elle modifiée ? Certaines études ont déjà abordé cette problématique (Greer et Yan, 2010 ; 2011 ; Mitchelstein et Boczkowski, 2009), mais elles se sont concentrées principalement sur des analyses quantitatives de l'offre et sur le rapport entre le journal et le lecteur plutôt que sur l'information en elle-même.

Notre étude se concentrera sur les journaux quotidiens français. À partir de l'analyse de la structure de l'information et de l'organisation du contenu proposé sur chaque support, nous reconstruirons les stratégies de définition du contenu du journal. Nous chercherons à distinguer les facteurs dérivant des choix rédactionnels de ceux qui sont déterminés par des contraintes techniques. Les changements opérés ont-ils été décidés dans le cadre de la définition d'une nouvelle architecture de l'information ou ne sont-ils que la conséquence d'adaptations ponctuelles de la stratégie en réponse à des besoins techniques liés aux différentes plateformes.

\section{Les quotidiens face aux nouveaux médias}

Les nouvelles technologies ont changé les pratiques de travail des journalistes avant même l'apparition du Web 2.0. Boczkowski dans son ouvrage fondamental Digitizing the news (2004a) a noté des innovations dans les mécanismes de rédaction dès 1998. De nombreux auteurs ont étudié ces transformations et ont cherché à évaluer le rôle des technologies et à analyser leurs effets sur le travail des journalistes (Maeyer, 2010 ; Rebillard, 2007). Sans entrer dans le détail de la discussion entre déterminisme et constructivisme technologique (Domingo, 2008), entre révolution technologique (Ortoleva, 2009) et mythe du journalisme (Maeyer, 2010), notre analyse part de l'état des lieux du journalisme en ligne pour aborder le sujet de l'organisation de l'information.

Aujourd'hui tous les journaux français ont une version en ligne (Charon et Le Floch, 2011). Dans une première phase «publier, c'était transférer le journal imprimé sur l'internet» (Boczkowski, 2010, 48), observait un des journalistes de Clarin.com interviewé par Boczkowski dans son étude sur les pratiques rédactionnelles du 
web argentin. Suite à la crise liée à la bulle internet, les journaux sont obligés de trouver de nouvelles formes de rentabilité ${ }^{3}$ et commencent à réfléchir de manière plus approfondie aux moyens d'adapter leur offre au nouveau média. Ils distinguent, par exemple, des modes de production des actualités selon qu'elles sont « froides » ou «chaudes» (Boczkowski, 2010). Ils intègrent interactivité et multimédia dans leur offre (Boczkowski, 2004b). Les médias de la presse écrite doivent tous désormais se demander comment associer au journal papier une offre électronique riche et variée (Charon, 2010).

Les premiers outils numériques proposés sont les flux RSS et les newsletters. Les quotidiens offrent leurs articles sous forme de syndication, c'est-à-dire à travers un flux RSS librement accessible et téléchargeable (Gill, 2005 ; Grossnickle et al., 2005). Par ailleurs, la newsletter, qui était initialement une sélection d'actualités adressée au lecteur par email, devient aujourd'hui un service bien plus complexe lié à la création d'un compte sur le site web du journal et à la possibilité de choisir des services personnalisés (Thurman et Schifferes, 2012). Plus récemment, les journaux se sont lancés sur les réseaux sociaux (Greer et Yan, 2010). Généralement, ils ouvrent une page sur Facebook et sur Google+ et un compte sur Twitter. Enfin, ils peuvent proposer une ou plusieurs applications gratuites ou payantes pour garantir à leurs lecteurs les dernières actualités en continu sur leurs smartphones et tablettes. Ceci impose à la rédaction du journal de proposer des actualités pour toutes ces différentes plateformes et de s'interroger sur le type de contenu à envoyer à chacune, sur sa forme et sa périodicité.

\section{Le cas des quotidiens français}

Afin de reconstruire les stratégies de contenu multiplateforme, nous avons mené une étude sur les journaux quotidiens français. L'échantillon est constitué par trois journaux nationaux (Le Monde, Le Figaro, Libération), un journal de la presse gratuite (20 Minutes), et deux journaux régionaux (Le Parisien et Ouest-France). Selon les

3 Depuis 2002 Le Monde offre un abonnement payant à la version électronique du journal. En 2005 le journal déclare que $40 \%$ de ses revenus sont issus de souscriptions (Attias, 2006, 147). 
données de l'OJD (Office de justification de la diffusion), il s'agit des six journaux quotidiens dont le site web reçoit le plus de visites (mai 2013).

L'analyse a été organisée en trois phases. Dans une première phase, nous avons comparé les versions électroniques et les flux RSS. Nous avons combiné une analyse quantitative des volumes d'information et une analyse qualitative du type d'information ${ }^{4}$. Ensuite, nous avons analysé l'offre sur les réseaux sociaux (Facebook, Google+ et Twitter) : le type de contenu proposé, sa forme et son adaptation à la plateforme. Enfin, à travers des entretiens semi-directifs avec des membres de la rédaction (quand c'était possible avec le responsable multimédia) ${ }^{5}$, nous avons cherché à reconstruire la stratégie de contenu sous-jacente à l'offre multiplateforme (Guyot, 2009).

\section{Résultats : l'offre multiplateforme}

Ces six journaux ont un site web qui offre une version en ligne du journal. Ils ont également une "édition abonnés », c'est-à-dire une offre payante qui permet d'accéder à la version numérique du journal papier et à certains contenus et services réservés aux abonnés. De ce point de vue, c'est Le Figaro qui propose l'offre la plus riche avec plusieurs contenus exclusifs. Seul 20 Minutes, en cohérence avec sa politique de gratuité (Rieffel, 2010), n'a pas d'offre payante.

En outre, tous ces quotidiens proposent des applications pour mobile/tablette ${ }^{6}$. En général, ces applications permettent d'accéder exactement à la même sélection d'actualités du site web, laquelle ne fait pas l'objet d'un travail éditorial d'adaptation du contenu. Récemment, certains journaux (dans notre échantillon tous à l'exception d'Ouest-France) ${ }^{7}$ ont ajouté la possibilité de recevoir des alertes «push». Cet outil est encore employé avec parcimonie :

$4 \quad$ L'analyse des flux RSS a été effectuée grâce à une base de données produite dans le cadre du projet ANR geomedia. Voir http://geomedia.hypotheses.org.

5 Nous avons réalisé vingt entretiens en contactant aussi des journalistes d'autres médias non inclus dans l'échantillon.

6 Le Figaro, Le Monde et Ouest-France offrent deux applications séparées, une gratuite et une payante.

7 L'application mobile de Ouest-France propose, quant à elle, un outil « reporter » pour envoyer des textes ou des photos à la rédaction. 
pendant la période observée 8 les journaux n'ont jamais envoyé plus de trois actualités par jour. Les mêmes alertes peuvent être reçues par email. La sélection de ces actualités implique évidement un travail rédactionnel.

En passant aux outils Web 2.0, on trouve une offre plus variée. Tous ont des fils RSS, des newsletters, la possibilité de créer un compte personnalisé, une page Facebook, un compte Twitter et un compte Google+, mais chacun trouve des solutions différentes pour adapter ses actualités aux plateformes. Le Parisien propose, en plus, un compte Pinterest ${ }^{9}$.

Pour ce qui concerne les flux RSS, 20 Minutes et Ouest-France ont un fil unique tandis que les autres journaux en ont plusieurs, en général un par rubrique. Le Parisien a aussi 9 fils locaux et Le Monde propose de nombreux fils thématiques. Dans tous les journaux considérés, les fils sont générés de façon automatique par le système de gestion du contenu du site web (Cabrolié, 2010) et ils incluent toutes les actualités de la page du site web correspondant à la rubrique ${ }^{10}$.

Un fil RSS est constitué par plusieurs items et chaque item par un titre et une description (Hmedeh et al., 2011). Le fil «à la Une », qui correspond à la page d'accueil, contient un nombre variable d'actualités entre 161 par semaine pour 20 Minutes et 623 pour Ouest-France 11 (tableau 1). De manière analogue, chaque item est constitué par un nombre variable de mots entre 70 pour $L e$ Figaro et 10 pour Ouest-France. Cette différence s'explique par le fait que Le Figaro, comme Libération, propose dans chaque item des «Articles en rapport» suggérés par le journaliste. Par ailleurs, les résumés du Monde, du Figaro et de Libération correspondent à

8 Les données présentées dans ce paragraphe sont basées sur une période d'observation des journaux de l'échantillon d'une durée d'un mois : du 13 mai au 9 juin 2013.

9 Réseau social online pour le partage de photos.

10 À travers les entretiens, nous avons pu identifier une seule exception, $L e$ Monde diplomatique. Dans ce cas, le fil RSS est produit par la rédaction. Cela permet de proposer les contenus d'un journal mensuel de manière quotidienne. Il faut mettre l'accent sur le fait que ce journal est le seul, de ceux considérés, où le responsable technique des supports web et médias est à la fois journaliste et documentaliste.

11 Nous faisons référence à la moyenne hebdomadaire parce que la fréquence des fils est très variable d'un jour à l'autre. Certains journaux comme Ouest-France n'envoient pas d'items le dimanche. 
l'abstract de l'article sur la page d'accueil et sont le résultat d'un travail rédactionnel (Le Figaro a une personne qui révise titres et résumés pour améliorer la performance du site web sur les moteurs de recherche) tandis que les résumés du Parisien et de 20 Minutes sont produits automatiquement par l'extraction des premiers mots de l'article. Enfin, les items de Ouest-France sont constitués seulement par le titre.

\begin{tabular}{|c|c|c|}
\hline Titre & $\begin{array}{c}\text { Moyenne hebdomadaire } \\
\text { d'items }\end{array}$ & $\begin{array}{c}\text { Moyenne de mots par } \\
\text { item }\end{array}$ \\
\hline Le Monde à la Une & 491 & 40,5 \\
\hline Le Figaro à la Une & 475 & 70 \\
\hline Le Parisien à la Une & 220 & 41,5 \\
\hline Libération à la Une & 326 & 56 \\
\hline 20 Minutes à la Une & 161 & 10 \\
\hline Ouest-France & 623 & \\
\hline
\end{tabular}

Tableau 1 : Structure des flux RSS par journal.

Pour ce qui concerne les newsletters, même si chaque journal a une politique spécifique, on peut identifier un trait commun : la proposition d'une newsletter générale gratuite qui correspond à la Une du site (et par conséquent au fil RSS). En outre, Le Monde, Le Figaro et Libération distinguent des newsletters gratuites, qui coöncident avec des sections du site, et d'autres payantes. La guerre entre les newsletters ne se joue pas vraiment au niveau du contenu (qui est souvent le même que celui du site web), mais plutôt sur l'heure d'envoi. Le Monde garantit l'envoi de la Une à 8 h 30 et du journal électronique à $14 \mathrm{~h}$, Le Figaro envoie la Une la veille à $22 \mathrm{~h}$ et la newsletter quotidienne à $9 \mathrm{~h}$, Libération envoie une newsletter matinale à $9 \mathrm{~h}$, Le Parisien et Ouest-France à $10 \mathrm{~h}, 20$ Minutes à $5 \mathrm{~h} 30$ et à $13 \mathrm{~h}$. L'inscription aux newsletters peut être gérée par le compte personnalisé. Pour certains journaux, comme Le Monde et Ouest-France, le lecteur ne peut se servir du compte que pour 
gérer ses abonnements tandis que dans les autres quotidiens il peut commenter les articles.

Venons-en à considérer l'activité sur Twitter ${ }^{12}$ : chaque tweet peut n'être constitué que d'un texte court, qui généralement coïncide avec le titre de l'article sur le site, (Le Figaro, Libération et OuestFrance) ou peut inclure un résumé (Le Monde, Le Parisien et parfois 20 Minutes). En général ces comptes sont employés pendant la journée (ils sont interrompus entre $23 \mathrm{~h}$ et $6 \mathrm{~h}$ ) par les journalistes pour signaler des articles de chacune des rubriques. Le flux de tweets peut être très variable parce qu'il dépend d'une contribution manuelle de la rédaction. Les mêmes articles peuvent être «tweetés » plusieurs fois s'ils sont mis à jour. De plus, on peut parfois trouver des re-tweets via les comptes personnels des journalistes ou les comptes des rubriques et des magazines du quotidien. Il résulte d'une telle variété qu'il n'y a pas de correspondance (et souvent même pas de similarité) entre les flux Twitter et les contenus proposés sur les autres plateformes, et qu'il est également difficile de retrouver une politique claire et cohérente dans ce flux d'éléments.

L'offre sur Facebook (voir tableau 2) est encore plus différenciée que sur Twitter. Dans la sélection des contenus, une attention particulière est donnée au type d'objet publié (vidéos et photos sont privilégiées) et au sujet, qui appartient souvent à la catégorie des « curiosités ». La périodicité de publication est très variable: les messages peuvent concerner des histoires du jour même, du jour précédent ou encore des histoires qui seront publiées le jour suivant en papier. Le nombre de mentions « j'aime » figurant dans les pages du Parisien, du Monde et du Figaro montre qu'elles sont bien plus appréciées que celles des autres journaux. Dans le cas du Monde, qui a pourtant une des pages les moins riches (avec en moyenne 4-5 messages par jour), les lecteurs participent activement par des commentaires, par la mention «j'aime » et le partage de contenu. Ces pages sont également celles où les textes montrent le moins de travail rédactionnel. Souvent, on trouve exactement le même résumé que sur Twitter ou le flux RSS. Cela signifie que le travail de la rédaction se concentre sur la sélection de contenus et non sur leur style. En général, ces rédactions privilégient des scoops potentiels (« dernière minute » ou «alerte » ou « exclusif »).

12 Nous avons considéré seulement le compte principal officiel sur les réseaux sociaux. 
Les pages de Libération, de Ouest-France et de 20 Minutes proposent des contenus avec un style clairement modifié pour rencontrer les attentes des lecteurs Facebook. Par exemple, OuestFrance ouvre la journée du 29 juin 2013 avec le message : «Crise oblige, de nombreux Français renoncent aux vacances. Et vous, aurez-vous la chance de partir cet été ?» quand le titre de l'article correspondant est «Vacances d'été. Moins d'un Français sur deux partira ». Le même jour, Libération publie le message suivant (sans un article correspondant): «Puisque nous sommes entre nous ce week-end et qu'on va pouvoir allumer la télé incessamment sous peu pour regarder le Tour, quels sont vos plus grands souvenirs de cette épreuve cycliste ?». Il faut noter que, dans les deux cas, peu de lecteurs répondent. Le Figaro a adopté une position intermédiaire. La plupart des contenus du journal sont repris par le site, mais on peut trouver parfois des contenus adaptés au style du réseau.

Plusieurs journaux proposent des messages en ouverture et clôture de journée. Par exemple, Le Figaro publie tous les jours un message d'ouverture («Bonjour à tous, voici la Une du vendredi 14 juin» vers $9 \mathrm{~h}$ ) et de clôture de journée («Bonsoir! Voici le résumé des informations de la journée » vers $17 \mathrm{~h}$ ).

\begin{tabular}{|c|c|c|c|c|}
\hline Titre & $\begin{array}{c}\text { Messages par } \\
\text { jour }\end{array}$ & $\begin{array}{c}\text { «Hashtag } \\
\text { de Facebook }\end{array}$ & $\begin{array}{c}\text { Mention } \\
\text { «J'aime » }\end{array}$ & $\begin{array}{c}\text { «Personnes } \\
\text { en parlent } 13\end{array}$ \\
\hline Le Monde & $0-8$ & Oui & 577438 & 59083 \\
\hline Le Figaro & $23-30$ & Non & 584466 & 50812 \\
\hline Le Parisien & $14-52$ & Oui & 835935 & 78891 \\
\hline Libération & $8-10$ & Non & 144402 & 16024 \\
\hline 20 Minutes & $15-18$ & Non & 170078 & 16403 \\
\hline Ouest-France & $2-5$ & Non & 28965 & 4213 \\
\hline
\end{tabular}

Tableau 2 : Offre Facebook par journal.

13 Il s'agit du nombre de membres Facebook ayant interagi avec la page pendant les 7 derniers jours. 
Pour ce qui concerne Google+, cet outil n'est pas très développé. Toutes les rédactions sentent l'obligation d'avoir une page, mais elles ne voient pas suffisamment d'intérêt chez les lecteurs pour investir sur l'adaptation des contenus à cette plateforme. Les rédactions publient sur Google+ une sélection ou tous les contenus publiés sur Facebook.

\section{Discussion : la place de l'architecture de l'information}

Cette analyse montre la différenciation de l'offre d'un journal à l'autre et fait soupçonner la complexité du travail au niveau rédactionnel. À plusieurs reprises, nous avons observé qu'une rédaction peut proposer exactement la même actualité sur plusieurs plateformes avec simplement parfois de petites différences. C'est le cas notamment des fils RSS, des newsletters, des actualités sur mobile et des alertes. On a vu que la Une des newsletters peut être reprise également sur Facebook. Par ailleurs, les contenus sur les réseaux sociaux (principalement sur Twitter) sont très variés et il est parfois difficile d'identifier la stratégie mise en œuvre. Les entretiens que nous avons conduits nous ont permis de comprendre les mécanismes cachés derrière une telle distribution de l'information entre une plateforme et l'autre.

Nous pouvons identifier deux types d'organisation: une organisation qu'on pourrait définir comme formalisée, qu'on retrouve essentiellement au Figaro, et une organisation plus informelle. Le directeur des opérations au Figaro, Thomas Doduik, a été le seul à se montrer à l'aise avec le concept d'architecture de l'information. L'offre multiplateforme du Figaro suit une politique précise, définie par un code écrit et par des normes établies au niveau central. Un seul journaliste prépare et adapte l'actualité aux différentes plateformes (du papier au web et aux réseaux sociaux) selon ces normes ${ }^{14}$. Ensuite, l'actualité n'est révisée que par un spécialiste $\mathrm{SEO}^{15}$ qui intervient sur les titres et sur la structure de la page d'accueil du journal pour répondre aux besoins du marketing. Une telle structure formalisée est également garantie par la forte automatisation dans la publication du contenu multiplateforme. Le même contenu est

14 La sélection des actualités pour chaque plateforme est faite de manière concertée pendant la réunion journalière de la rédaction.

15 Optimisation pour les moteurs de recherche (en anglais, Search engine optimization : SEO). 
publié sur presque toutes les plateformes. En effet, le système de gestion du contenu permet facilement d'inclure la même actualité sur le site web, dans le flux RSS, dans la newsletter, sur mobile et aussi sur Twitter. Cela est évident pour les alertes qui peuvent être reçues avec une forme identique par de nombreux canaux. Dans ce contexte, l'architecture de l'information devient un concept opératoire employé principalement pour répondre à l'exigence de rapidité et d'optimisation des ressources. Cette organisation de l'information, en effet, garantit au Figaro une production de contenu très riche sur toutes les plateformes avec un minimum de personnel.

Les entretiens conduits dans d'autres journaux ont permis de constater une attitude très différente à l'égard du concept d'architecture de l'information. En général, en réponse à la première question (l'offre multiplateforme du journal est-elle basée sur une architecture de l'information définie au niveau central par la rédaction?), le journaliste demandait qu'on reformule celle-ci. À plusieurs reprises, nous avons perçu un rejet du concept d'organisation de l'information et de l'idée de formalisation des méthodes de publication des articles. Florent Latrive, responsable multimédia de Libération, reconnaît l'existence d'une organisation centralisée de l'information «parce que sont les mêmes personnes qui pilotent l'information proposée sur les différents supports », mais il refuse l'idée d'une offre formalisée qui répondrait à des règles spécifiques.

Plusieurs journalistes ont mis l'accent sur l'importance de l'audience. L'information doit être adaptée aux intérêts des lecteurs et la rédaction ne dispose pas d'assez d'information sur les lecteurs de ces nouvelles plateformes pour définir des normes précises et toujours valables. C'est pour cela que dans la plupart des rédactions la gestion de Twitter et Facebook peut beaucoup varier. Ces plateformes sont conçues comme un terrain de bricolage (Comtet, 2009) et d'expérimentation. Florent Latrive conclut l'entretien en disant: «L'offre n'est pas formalisée et il n'y a pas de règles spécifiques. Elle change sur la base du retour de l'audience. L'équipe discute et décide quoi proposer... Cela correspond à comment marche un journal en général ». Dans ce dernier contexte le concept d'architecture de l'information non seulement n'est pas mis en œuvre, mais il peut même être considéré comme dangereux pour la liberté d'expression du journaliste. 


\section{Conclusion}

L'observation de l'offre multiplateforme de ces six quotidiens français a permis de confirmer que le passage de la version papier à la version électronique d'un journal n'est pas une simple migration du contenu d'un support à l'autre (Boczkowski, 2010 ; Adams, 2007 ; Nguyen, 2008). De plus, l'arrivée du Web 2.0 a obligé les rédactions à proposer leurs actualités sur plusieurs plateformes et à s'interroger sur le contenu à envoyer, sa forme et sa périodicité, pour chacune d'entre elles. À la nouveauté des outils, s'ajoutent plusieurs problèmes pour le journaliste: le manque d'information sur les usagers de chaque plateforme et sur leurs usages qui rend difficile l'adaptation de l'information; et la rapidité de la mise à jour exigée par ces plateformes (Charon, 2010). Aussi le journaliste doit-il produire plus de contenu en moins de temps et à moindre prix ${ }^{16}$ (Bakker, 2012, 628).

Après avoir décrit l'offre de contenu de chaque journal, nous sommes passés à l'analyse de l'organisation de la rédaction (Guyot, 2006). Nous avons cherché à voir comment les rédactions gèrent cette différenciation des contenus et, notamment, si la stratégie de différenciation est définie par une charte centralisée et pilotée par une personne dans la rédaction ou si elle est le produit du travail conjoint de l'équipe des journalistes. Dans les entretiens, nous avons fait référence à plusieurs reprises au concept d'architecture de l'information pour voir s'il était intervenu dans la réflexion de la rédaction pour construire la nouvelle offre d'actualités.

Cette étude s'est révélée très intéressante parce qu'elle a permis d'identifier deux réponses opposées à cette problématique. Dans un cas, le concept d'architecture de l'information est perçu comme positif et constructif et dans l'autre, au contraire, comme négatif et dangereux. Cette constatation ouvre plusieurs pistes de recherche future. D'abord, il sera intéressant de voir comment les offres émanant de journaux différemment organisés sont perçues par l'audience : notamment comment les lecteurs perçoivent les essais rédactionnels sur Facebook et Twitter. En outre, il pourrait être utile d'élargir le terrain d'étude à d'autres organes de presse français, notamment les hebdomadaires qui, face au Web 2.0, sont confrontés

16 Bakker (2012) analyse de manière très efficace les nouvelles modalités de journalisme moins coûteuses : « low-paid », « no-paid », « automated content ». 
en plus au problème de la périodicité des contenus, et également au niveau international, pour voir si l'offre multiplateforme présente des caractéristiques spécifiques selon les pays.

MARTA SEVERO

Laboratoire GERiiCO - Université de Lille 3

\section{BIBLIOGRAPHIE}

Adams J. W., (2007), U.S. Weekly Newspapers Embrace Web Sites, in Newspaper Research Journal, vol. 28, n 4, pp. 36-50.

Albert P., (2008), La presse française, Paris, La Documentation française, 215 p.

Attias D., (2006), Quel modèle économique pour la presse sur Internet?, in Le Temps des médias, 2006/1, n 6, pp. 143-150.

Bakker P., (2012), Aggregation, content farms and huffinization, in Journalism Practice, $n^{\circ}$ 5-6, pp. 627-637.

Boczkowski P. J., (2004a), Digitizing the news : innovation in online newspapers, Cambridge Mass., MIT Press, 243 p.

Boczkowski P. J., (2004b), The Processes of Adopting Multimedia and Interactivity in Three Online Newsrooms, in Journal of Communication, ${ }^{\circ}$ 54, pp. 197-213.

Boczkowski P. J., (2010), Ethnographie d'une rédaction en ligne argentine. Les logiques contraires de la production de l'information chaude et froide, in Réseaux, vol. 2, n 160-161, pp. 43-78.

Cabrolié S., (2010), Les journalistes du parisien.fr et le dispositif technique de production de l'information, in Réseaux, $\mathrm{n}^{\circ} 160$, pp. 79-100.

Charon J.-M., (2010), De la presse imprimée à la presse numérique, in Réseaux, vol. $2, \mathrm{n}^{\circ} 160-161$, pp. $255-281$.

Charon J.-M. et Le Floch P., (2011), La presse en ligne, Paris, La Découverte, coll. « Repères », 128 p.

Comtet I., (2009), Entre usage professionnel des TIC et structure organisationnelle : la capacité au bricolage comme compétence adaptative, in Études de communication, $\mathrm{n}^{\circ} 33$, pp. 119-134.

Deuze M., (2003), The Web and its Journalisms: Considering the Consequences of Different Types of News media Online, in New Media \& Society, vol. 2, $\mathrm{n}^{\circ} 5$, pp. 203-230.

Domingo D., (2008), Interactivity in the daily routines of online newsrooms: dealing with an uncomfortable myth, in Journal of Computer-Mediated Communication, vol. 13, n 3, pp. 680-704.

Éveno P., (2008), La presse quotidienne nationale, fin de partie ou renouveau?, Paris, Vuibert, 219 p.

Gentzkow M. A., (2004), Valuing new goods in a Model with Complementarity: Online Newspapers, American Economic Review, vol. 97, n 3, pp. 713-744.

Gill K., (2005), Blogging, RSS and the Information Landscape: A Look At Online News, in WWW2005, http://faculty.washington.edu/kegill/pub/gill_www2005_rss. pdf, date de la dernière visite : 15 juin 2013. 
Greer J. et Yan Y., (2011), Newspapers Connect with Readers Through Multiple Digital Tools, in Newspaper Research Journal, vol. 32, n 4, pp. 83-97.

Greer J. et Yan Y., (2010), New ways of connecting with readers: How community newspapers are using Facebook, Twitter and other tools to deliver the news, in Grassroots Editor, vol. 51, n 4, pp. 1-7.

Grossnickle J., Board T., Pickens V. et Bellmont M., (2005), RSS - Crossing into the Mainstream, White paper, $12 \mathrm{p}$.

Guyot B., (2009), Se mouvoir au sein du monde de l'information: comment les personnes parlent de leur activité d'information, in Études de communication, $\mathrm{n}^{\circ} 33$, pp. 101-118.

Guyot B., (2006), Dynamiques informationnelles dans les organisations, Paris, Hermès-sciences, $234 \mathrm{p}$.

Hmedeh Z., Travers N., Vouzoukidou N., Christophides V., du Mouza C. et Scholl M., (2011), «Everything you would like to know about RSS feeds and you afraid to ask »,BDA'11, Base de Données Avancées Morocco.

Maeyer (de) J., (2010), « Être journaliste dans un environnement 2.0 », Les Cahiers du numérique, vol. 6, $\mathrm{n}^{\circ} 1$, pp. 157-177.

Mitchelstein E. et Boczkowski P. J., (2009), Between tradition and change. A review of recent research on online news production, in Journalism, vol. 10, $\mathrm{n}^{\circ} 5$, pp. 562586.

Nguyen A., (2008), Fabulous Monster: The Traditional Media's Fear-driven Innovation Culture in the Development of Online News, in Journalism Studies, vol. 9, $\mathrm{n}^{\circ} 1$, pp. 91-104.

Ortoleva P., (2009), Modern Mythologies, the Media and the Social Presence of Technology, in Observatorio (OBS*), vol. 3, $\mathrm{n}^{\circ}$ 1, pp. 1-12.

O'Reilly T., (2005), What is Web 2.0? Design Patterns and Business Models for the Next Generation of Software, http://www.oreillynet.com/lpt/a/6228, date de la dernière visite : 15 juin 2013.

Pew Internet et American Life Project, (2010), Understanding the Participatory News Consumer, http://www.journalism.org/analysis_report/understanding_ participatory_news_consumer, date de la dernière visite : 15 juin 2013.

Pisani F., (2009), Mort des journaux ou du journalisme?, http://pisani.blog.lemonde. fr/2009/04/04/mort-des-journaux-ou-du-journalisme/, date de la dernière visite : 15 juin 2013.

Poulet B., (2009), La fin des journaux et l'avenir de l'information, Paris, Gallimard, $210 \mathrm{p}$.

Rebillard F., (2007), Le web 2.0 en perspective : Une analyse socio-économique de l'Internet, Paris, L'Harmattan, $162 \mathrm{p}$.

Rieffel R., (2010), Mythologie de la presse gratuite, Le Cavalier Bleu, 96 p.

Rosenfeld L. et Morville P., (1998), Information architecture for the World Wide Web. 1st. Sebastopol, CA: O'Reilly \& Associates. ISBN 0-596-52734-9.

Schwartzenberg E., (2007), Spéciale dernière. Qui veut la mort de la presse quotidienne française ?, Paris, Calmann-Lévy, 281 p.

Tarlé (de) A., (2006), Presse et Internet. Une chance, un défi : enjeux économiques, enjeux démocratiques, in Cahier En temps réel, $\mathrm{n}^{\circ} 26,22 \mathrm{p}$.

Thurman N. et Schifferes S., (2012), The future of personalization at news websites the future of personalization at news websites, in Lessons from a longitudinal study. Journalism Studies, vol. 13, n 5-6, pp. 775-790. 